\title{
Solving the Axisymmetric Inverse Heat Conduction Problem by a Wavelet Dual Least Squares Method
}

\author{
Wei Cheng' and Chu-Li Fu² \\ ${ }^{1}$ College of Science, Henan University of Technology, Zhengzhou 450001, China \\ ${ }^{2}$ School of Mathematics and Statistics, Lanzhou University, Lanzhou 730000, China \\ Correspondence should be addressed to Chu-Li Fu, fuchuli@lzu.edu.cn \\ Received 17 August 2008; Revised 23 January 2009; Accepted 10 March 2009 \\ Recommended by Ugur Abdulla
}

We consider an axisymmetric inverse heat conduction problem of determining the surface temperature from a fixed location inside a cylinder. This problem is ill-posed; the solution (if it exists) does not depend continuously on the data. A special project method-dual least squares method generated by the family of Shannon wavelet is applied to formulate regularized solution. Meanwhile, an order optimal error estimate between the approximate solution and exact solution is proved.

Copyright (C) 2009 W. Cheng and C.-L. Fu. This is an open access article distributed under the Creative Commons Attribution License, which permits unrestricted use, distribution, and reproduction in any medium, provided the original work is properly cited.

\section{Introduction}

Inverse heat conduction problems (IHCP) have become an interesting subject recently, and many regularization methods have been developed for the analysis of IHCP [1-13]. These methods include Tikhonov method [1, 2], mollification method [3, 4], optimal filtering method [5], lines method [6], wavelet and wavelet-Galerkin method [7-11], modified Tikhonov method [12] and "optimal approximations" [13], and so forth. However, most analytical and numerical methods were only used to dealing with IHCP in semiunbounded region. Some works of numerical methods were presented for IHCP in bounded domain [1419].

Chen et al. [14] applied the hybrid numerical algorithm of Laplace transform technique to the IHCP in a rectangular plate. Busby and Trujillo [15] used the dynamic programming method to investigate the IHCP in a slab. Alifanov and Kerov [16] and Louahlia-Gualous et al. [17] researched IHCP in a cylinder. However to the authors' knowledge, most of them did not give any stability theory and convergence proofs.

In this paper, we will treat with a special IHCP whose physical model consists of an infinitely long cylinder of radius $R$. It is considered axisymmetric and a thermocouple 
(measurement equipment of temperature) is installed inside the cylinder (at the radius $r_{1}$, $0<r_{1}<R$ ). The correspondingly mathematical model of our problem can be described by the following axisymmetric heat conduction problem:

$$
\begin{gathered}
\frac{\partial u}{\partial t}=\Delta u=\frac{\partial^{2} u}{\partial r^{2}}+\frac{1}{r} \frac{\partial u}{\partial r}, \quad 0<r \leq R, t>0, \\
u(r, 0)=0, \quad 0 \leq r \leq R \\
u\left(r_{1}, t\right)=g(t), \quad t \geq 0 \\
u(r, t) \quad \text { bounded in } r=0, t>0
\end{gathered}
$$

where the functions $u(r, \cdot)$ and $g(\cdot)$ belong to $L^{2}(0, \infty)$ for every fixed $r \in(0, R), r$ is the radial coordinate, $g(t)$ denotes the temperature history at one fixed radius $r_{1}\left(0<r_{1}<R\right)$ of cylinder. We want to recover $u(r, \cdot)$ for $r_{1}<r \leq R$. This problem is ill-posed problem; a small perturbation in the data may cause dramatically large errors in the solution $u(r, \cdot)$ (The details can be seen in Section 2).

To the authors' knowledge, up to now, there is no regularization theory with error estimate for problem (1.1) in the interval $r_{1}<r \leq R$. The major objective of this paper is to do the theoretic stability and convergence estimates for problem (1.1).

Xiong and Fu [11] and Regińska [20] solved the sideways heat equation in semiunbounded region by applying the wavelet dual least squares method, which is based on the family of Meyer wavelet. In this paper, we will apply a wavelet dual least squares method generated by the family of Shannon wavelet to problem (1.1) in bounded domain for determining surface temperature. According to the optimality results of general regularization theory, we conclude that our error estimate on surface temperature is order optimal.

\section{Formulation of Solution of Problem (1.1)}

As we consider problem $(1.1)$ in $L^{2}(\mathbb{R})$ with respect to variable $t$, we extend $u(r, \cdot), g(\cdot):=$ $u\left(r_{1}, \cdot\right), f(\cdot):=u(R, \cdot)$, and other functions of variable $t$ appearing in the paper to be zero for $t<0$. Throughout the paper, we assume that for the exact $g$ the solution $u$ exists and satisfies an apriori bound

$$
\|f(\cdot)\|_{p}:=\|u(R, \cdot)\|_{p} \leq E, \quad p \geq 0
$$

where $\|f(\cdot)\|_{p}$ is defined by

$$
\|f(\cdot)\|_{p}:=\left(\int_{-\infty}^{\infty}\left(1+\xi^{2}\right)^{p}|\widehat{f}(\xi)|^{2} d \xi\right)^{1 / 2}
$$

Since $g$ is measured by the thermocouple, there will be measurement errors, and we would actually have as data some function $g_{\delta} \in L^{2}(\mathbb{R})$, for which

$$
\left\|g_{\delta}(\cdot)-g(\cdot)\right\| \leq \delta
$$


where the constant $\delta>0$ represents a bound on the measurement error, and $\|\cdot\|$ denotes the $L^{2}(\mathbb{R})$ norm and

$$
\widehat{h}(\xi)=\frac{1}{\sqrt{2 \pi}} \int_{-\infty}^{\infty} e^{-i \xi t} h(t) d t
$$

is the Fourier transform of function $h(t)$. The problem (1.1) can be formulated, in frequency space, as follows:

$$
\begin{gathered}
i \xi \widehat{u}(r, \xi)=\frac{\partial^{2} \widehat{u}(r, \xi)}{\partial r^{2}}+\frac{1}{r} \frac{\partial \widehat{u}(r, \xi)}{\partial r}, \quad r \in(0, R], \xi \in \mathbb{R}, \\
\widehat{u}\left(r_{1}, \xi\right)=\widehat{g}(\xi), \quad \xi \in \mathbb{R}, \\
|\widehat{u}(0, \xi)|<\infty, \quad \xi \in \mathbb{R} .
\end{gathered}
$$

Then we have the following lemma.

Lemma 2.1. Problem (2.5)-(2.7) has the solution given by

$$
\widehat{u}(r, \xi)=\frac{I_{0}(\sqrt{i \xi} r)}{I_{0}\left(\sqrt{i \xi} r_{1}\right)} \widehat{g}(\xi), \quad r \in[0, R], \xi \in \mathbb{R},
$$

where $I_{0}(z)$ denotes modified spherical Bessel function which given by [21]

$$
I_{0}(z)=\sum_{k=0}^{\infty} \frac{1}{(k !)^{2}}\left(\frac{z}{2}\right)^{2 k}
$$

Proof. Due to [21], we can solve (2.5), in the frequency domain, to obtain

$$
\widehat{u}(r, \xi)=A(\xi) I_{0}(\sqrt{i \xi} r)+B(\xi) K_{0}(\sqrt{i \xi} r) \quad \xi \in \mathbb{R},
$$

where $K_{0}(z)$ denotes also modified spherical Bessel function which is given by

$$
K_{0}(z)=-I_{0}(z)\left(\ln \frac{z}{2}+C\right)+\sum_{k=1}^{\infty} \frac{1}{(k !)^{2}}\left(1+\frac{1}{2}+\cdots+\frac{1}{k}\right)\left(\frac{z}{2}\right)^{2 k} .
$$

Combining $\lim _{z \rightarrow 0} K_{0}(z)=\infty$ with condition(2.7), we obtain $B(\xi)=0$, that is,

$$
\widehat{u}(r, \xi)=A(\xi) I_{0}(\sqrt{i \xi} r), \quad r \in[0, R], \xi \in \mathbb{R} .
$$

According to [21], there holds

$$
\left|I_{0}(\sqrt{i \xi} r)\right|=|\operatorname{ber}(\sqrt{|\xi| r})+i \sigma \operatorname{bei}(\sqrt{|\xi| r})|=\left[\sum_{k=0}^{\infty} \frac{(\sqrt{|\xi| r / 2})^{4 k}}{(k !)^{2}(2 k) !}\right]^{1 / 2},
$$


where $\sigma=\operatorname{sgn}(\xi)$, both $\operatorname{ber}(x)$ and bei $(x)$ denote the Kelvin functions. Since $0 \leq r \leq R,|\xi| \geq 0$, we have

$$
\sum_{k=0}^{\infty} \frac{(\sqrt{|\xi|} / 2)^{4 k}}{(k !)^{2}(2 k) !}=1+\frac{(\sqrt{|\xi|} / 2)^{8}}{(2 !)^{2}(4) !}+\frac{(\sqrt{|\xi|} / 2)^{12}}{(3 !)^{2}(6) !}+\cdots \geq 1
$$

Therefore, for $0 \leq r \leq R, \xi \in \mathbb{R}$,

$$
\left|I_{0}(\sqrt{i \xi} r)\right|==\left[\sum_{k=0}^{\infty} \frac{(\sqrt{|\xi|} / 2)^{4 k}}{(k !)^{2}(2 k) !}\right]^{1 / 2} \geq 1
$$

Solving the systems (2.6) and (2.12) using (2.15) we get

$$
A(\xi)=I_{0}^{-1}\left(\sqrt{i \xi} r_{1}\right) \hat{g}(\xi)
$$

Substitution of $A(\xi)$ in (2.16) into (2.12), we obtain (2.8).

Applying an inverse Fourier transform to (2.8), problem (1.1) has the solution

$$
u(r, t)=\frac{1}{\sqrt{2 \pi}} \int_{-\infty}^{\infty} e^{i \xi t} \frac{I_{0}(\sqrt{i \xi} r)}{I_{0}\left(\sqrt{i \xi} r_{1}\right)} \widehat{g}(\xi) d \xi, \quad(r, t) \in[0, R] \times \mathbb{R} .
$$

In order to obtain ill-posedness of problem (1.1) for $(r, t) \in\left(r_{1}, R\right] \times \mathbb{R}$, we need the following lemma.

Lemma 2.2. If function $\left|I_{0}(\sqrt{i \xi} r)\right|$ satisfies (2.15), then there exist positive constants $c_{k}, k=$ $1,2,3,4$, such that, for $r \in\left(r_{1}, R\right]$

$$
\begin{aligned}
& c_{1} \exp \left[\sqrt{|\xi| / 2}\left(r-r_{1}\right)\right] \leq\left|\frac{I_{0}(\sqrt{i \xi} r)}{I_{0}\left(\sqrt{i \xi} r_{1}\right)}\right| \leq c_{2} \exp \left[\sqrt{|\xi| / 2}\left(r-r_{1}\right)\right], \quad \xi \in \mathbb{R}, \\
& c_{3} \exp [\sqrt{|\xi| / 2}(r-R)] \leq\left|\frac{I_{0}(\sqrt{i \xi} r)}{I_{0}(\sqrt{i \xi} R)}\right| \leq c_{4} \exp [\sqrt{|\xi| / 2}(r-R)], \quad \xi \in \mathbb{R} .
\end{aligned}
$$

Proof. First, due to [21] and (2.15), we have, for $r \in\left[r_{1}, R\right]$ and $|\xi| \rightarrow \infty$,

$$
\left|I_{0}(\sqrt{i \xi} r)\right|=\left[\operatorname{ber}^{2}(\sqrt{|\xi| r})+\operatorname{bei}^{2}(\sqrt{|\xi| r})\right]^{1 / 2}=\frac{\exp [\sqrt{|\xi| / 2} r]}{\sqrt{2 \pi r \sqrt{|\xi|}}}\left[1+O\left(\frac{1}{|\xi|}\right)\right]
$$


then there exist positive constants $\widetilde{c}_{k}, k=1,2,3,4$, such that, for $|\xi|$ large enough, say $|\xi| \geq \xi_{0}$

$$
\begin{gathered}
\tilde{c}_{1} \frac{\exp [\sqrt{|\xi| / 2} r]}{\sqrt{2 \pi r \sqrt{|\xi|}}} \leq\left|I_{0}(\sqrt{i \xi} r)\right| \leq \tilde{c}_{2} \frac{\exp [\sqrt{|\xi| / 2} r]}{\sqrt{2 \pi r \sqrt{|\xi|}}}, \quad r \in\left(r_{1}, R\right], \\
\tilde{c}_{3} \frac{\exp \left[\sqrt{|\xi| / 2} r_{1}\right]}{\sqrt{2 \pi r_{1} \sqrt{|\xi|}}} \leq\left|I_{0}\left(\sqrt{i \xi} r_{1}\right)\right| \leq \tilde{c}_{4} \frac{\exp \left[\sqrt{|\xi| / 2} r_{1}\right]}{\sqrt{2 \pi r_{1} \sqrt{|\xi|}}} .
\end{gathered}
$$

From these we know that there exist positive constants $\widetilde{c}_{5}$ and $\widetilde{c}_{6}$ such that, for $r \in\left(r_{1}, R\right]$ and $|\xi| \geq \xi_{0}$,

$$
\tilde{c}_{5} \exp \left[\sqrt{|\xi| / 2}\left(r-r_{1}\right)\right] \leq\left|\frac{I_{0}(\sqrt{i \xi} r)}{I_{0}\left(\sqrt{i \xi} r_{1}\right)}\right| \leq \tilde{c}_{6} \exp \left[\sqrt{|\xi| / 2}\left(r-r_{1}\right)\right]
$$

Then, since function $\left|I_{0}(\sqrt{i \xi} r) / I_{0}\left(\sqrt{i \xi} r_{1}\right)\right|$ is continuous in the closed region $\left(r_{1}, R\right] \times\left[-\xi_{0}, \xi_{0}\right]$. Threrfore, there exist constants $\tilde{c}_{7}$ and $\tilde{c}_{8}$ such that, for $r \in\left(r_{1}, R\right]$ and $|\xi| \leq \xi_{0}$,

$$
\tilde{c}_{7} \exp \left[\sqrt{|\xi| / 2}\left(r-r_{1}\right)\right] \leq\left|\frac{I_{0}(\sqrt{i \xi} r)}{I_{0}\left(\sqrt{i \xi} r_{1}\right)}\right| \leq \tilde{c}_{8} \exp \left[\sqrt{|\xi| / 2}\left(r-r_{1}\right)\right] .
$$

Finally, combining inequalities (2.22) with (2.23), we can see that there exist others constants $c_{1}$ and $c_{2}$ such that, for $r \in\left(r_{1}, R\right]$, inequalities (2.18) are valid. Similarly, we obtain inequalities (2.19).

In order to formulate problem (1.1) for $r_{1}<r \leq R$ in terms of an operator equation in the space $X=L^{2}(\mathbb{R})$, we define an operator $K_{r}: u(r, \cdot) \mapsto g(\cdot)$, that is,

$$
\forall u(r, \cdot) \in X, \quad K_{r} u(r, t)=g(t), \quad r_{1}<r \leq R .
$$

From (2.8), we have

$$
\widehat{K_{r} u(r, \xi)}=\frac{I_{0}\left(\sqrt{i \xi} r_{1}\right)}{I_{0}(\sqrt{i \xi} r)} \widehat{u}(r, \xi)=\widehat{g}(\xi)
$$

Denote $\widehat{K_{r} u(r, \xi)}:=\widehat{K_{r}} \widehat{u}(r, \xi)$, and we can see that $\widehat{K}_{r}: L^{2}(\mathbb{R}) \mapsto L^{2}(\mathbb{R})$ is a multiplication operator:

$$
\widehat{K_{r}} \widehat{u}(r, \xi)=\frac{I_{0}\left(\sqrt{i \xi} r_{1}\right)}{I_{0}(\sqrt{i \xi} r)} \widehat{u}(r, \xi)
$$

From (2.26), we can prove the following lemma. 
Lemma 2.3. Let $K_{r}^{*}$ be the adjoint to $K_{r}$, then $K_{r}^{*}$ corresponds to the following problem where the left-hand side $\partial u / \partial t$ of problem (1.1) is replaced by $-\partial U / \partial t$, says

$$
\begin{gathered}
-\frac{\partial U}{\partial t}=\Delta U=\frac{\partial^{2} U}{\partial r^{2}}+\frac{1}{r} \frac{\partial U}{\partial r}, \quad 0<r \leq R, t \geq 0, \\
U(r, 0)=0, \quad 0 \leq r \leq R, \\
U\left(r_{1}, t\right)=g(t), \quad t \geq 0, \\
U(r, t) \quad \text { bounded in } r=0, t>0, \\
\widehat{K_{r}^{*}}=\frac{\overline{I_{0}\left(\sqrt{i \xi} r_{1}\right)}}{\overline{I_{0}(\sqrt{i \xi} r)}} .
\end{gathered}
$$

Proof. Via the the following relations, combining with (2.26),

$$
\left\langle K_{r} u, v\right\rangle=\left\langle\widehat{K_{r}} \widehat{u}, \widehat{v}\right\rangle=\left\langle\widehat{u}, \widehat{K}_{r}^{*} \widehat{v}\right\rangle=\left\langle u, K_{r}^{*} v\right\rangle=\left\langle\widehat{u}, \widehat{K_{r}^{*}} \widehat{v}\right\rangle
$$

we can get the adjoint operator $K_{r}^{*}$ of $K_{r}$ in frequency domain

$$
\widehat{K}_{r}^{*}=\widehat{K}_{r}^{*}=\frac{\overline{I_{0}\left(\sqrt{i \xi} r_{1}\right)}}{\overline{I_{0}(\sqrt{i \xi} r)}}
$$

On the other hand, the problem (2.27) can be formulated, in frequency space, as follows:

$$
\begin{gathered}
-i \xi \widehat{U}(r, \xi)=\frac{\partial^{2} \widehat{U}(r, \xi)}{\partial r^{2}}+\frac{1}{r} \frac{\partial \widehat{U}(r, \xi)}{\partial r}, \quad r \in(0, R], \xi \in \mathbb{R}, \\
\widehat{U}\left(r_{1}, \xi\right)=\widehat{g}(\xi), \quad \xi \in \mathbb{R}, \\
|\widehat{U}(0, \xi)|<\infty, \quad \xi \in \mathbb{R} .
\end{gathered}
$$

Taking the conjugate operator for problem $(2.5)-(2.7)$, we realize that $\widehat{U}(r, \xi)=\overline{\widehat{u}(r, \xi)}$. Therefore, by Lemma 2.1, we conclude that

$$
\widehat{U}(r, \xi)=\overline{\widehat{u}(r, \xi)}=\frac{\overline{I_{0}(\sqrt{i \xi} r)}}{\overline{I_{0}\left(\sqrt{i \xi} r_{1}\right)}} \widehat{g}(\xi),
$$


that is,

$$
\widehat{g}(\xi)=\frac{\overline{I_{0}\left(\sqrt{i \xi} r_{1}\right)}}{\overline{I_{0}(\sqrt{i \xi} r)}} \widehat{U}(r, \xi)=\widehat{K_{r}^{*}} \widehat{U}(r, \xi):=\widehat{K_{r}^{*} U}
$$

Hence the conclusion of Lemma 2.3 is proved. holds

The Parseval formula for the Fourier transform together with inequality (2.18), there

$$
\begin{aligned}
\|u(r, \cdot)\|^{2} & =\|\widehat{u}(r, \cdot)\|^{2} \\
& =\int_{-\infty}^{\infty}|\widehat{u}(r, \cdot)|^{2} d \xi \\
& =\int_{-\infty}^{\infty}\left|\widehat{g}(\xi) e^{\left(r-r_{1}\right) \sqrt{|\xi| / 2}}\right|^{2}\left|\frac{I_{0}(\sqrt{i \xi} r)}{I_{0}\left(\sqrt{i \xi} r_{1}\right)}\right|^{2} d \xi \\
& \geq c_{1}^{2} \int_{-\infty}^{\infty}\left|\widehat{g}(\xi) e^{\left(r-r_{1}\right) \sqrt{|\xi| / 2}}\right|^{2} d \xi .
\end{aligned}
$$

This implies that $\widehat{g}(\xi)$, which is Fourier transform of exact data $g(t)$, must decay rapidly at high frequencies since $r_{1}<r$. But such a decay is not likely to occur in the Fourier transform of the measured noisy data $g_{\delta}(t)$ at $r=r_{1}$. So, small perturbation of $g(t)$ in high frequency components can blow up and completely destroy the solution $u(r, t)$ given by $(2.17)$ for $r \in$ $\left(r_{1}, R\right]$.

\section{Wavelet Dual Least Squares Method}

\subsection{Dual Least Squares Method}

A general projection method for the operator equation $K u=g, K: X=L^{2}(\mathbb{R}) \mapsto X=L^{2}(\mathbb{R})$ is generated by two subspace families $\left\{V_{j}\right\}$ and $\left\{Y_{j}\right\}$ of $X$ and the approximate solution $u_{j} \in V_{j}$ is defined to be the solution of the following problem:

$$
\left\langle K u_{j}, y\right\rangle=\langle g, y\rangle, \quad \forall y \in Y_{j}
$$

where $\langle\cdot, \cdot\rangle$ denotes the inner product in $X$. If $V_{j} \subset R\left(K^{*}\right)$ and subspaces $Y_{j}$ are chosen in such a way that

$$
K^{*} Y_{j}=V_{j}
$$

Then we have a special case of projection method known as the dual least squares method. If $\left\{\psi_{\lambda}\right\}_{\lambda \in \tilde{I}_{j}}$ is an orthogonal basis of $V_{j}$ and $y_{\lambda}$ is the solution of the equation

$$
K^{*} y_{\lambda}=k_{\lambda} \psi_{\lambda}, \quad\left\|y_{\lambda}\right\|=1
$$


then the approximate solution is explicitly given by the expression

$$
u_{j}=\sum_{\lambda \in \tilde{I}_{j}}\left\langle g, y_{\lambda}\right\rangle \frac{1}{k_{\lambda}} \psi_{\lambda}
$$

\subsection{Shannon Wavelets}

In [22], the Shannon scaling function is $\phi=(\sin \pi t) / \pi t$ and its Fourier transform is

$$
\widehat{\phi}(\xi)= \begin{cases}1, & |\xi| \leq \pi \\ 0, & \text { otherwise }\end{cases}
$$

The corresponding wavelet function $\psi$ is given by its Fourier transform

$$
\widehat{\psi}(\xi)= \begin{cases}e^{-i(\xi / 2)}, & \pi \leq|\xi| \leq 2 \pi \\ 0, & \text { otherwise. }\end{cases}
$$

Let us list some notation: $\phi_{j, k}(t):=2^{j / 2} \phi\left(2^{j} t-k\right), \psi_{j, k}(t):=2^{j / 2} \psi\left(2^{j} t-k\right), j, k \in \mathbb{Z}, \Psi_{-1, k}:=\phi_{0, k}$ and $\Psi_{l, k}:=\psi_{l, k}$ for $l \geq 0$, the index set

$$
\tilde{I}=\{\{j, k\}: j, k \in \mathbb{Z}\} \subset \mathbb{Z}^{2}, \quad \tilde{I}_{J}=\{\{j, k\}: j=-1,0, \ldots, J-1 ; k \in \mathbb{Z}\} \subset \mathbb{Z}^{2} .
$$

Because $V_{J}=V_{J-1} \oplus W_{J-1}=V_{J-2} \oplus W_{J-2} \oplus W_{J-1}=\cdots=V_{0} \oplus W_{1} \oplus \cdots \oplus W_{J-1}$, hence we can define the subspaces $V_{J}$

$$
V_{J}=\overline{\operatorname{span}\left\{\Psi_{\lambda}\right\}_{\lambda \in \tilde{I}_{J}}}
$$

Define an orthogonal projection $P_{J}: L^{2}(\mathbb{R}) \mapsto V_{J}$ :

$$
P_{J} \varphi=\sum_{\lambda \in \tilde{I}_{J}}\left\langle\varphi, \Psi_{\curlywedge}\right\rangle \Psi_{\curlywedge}, \quad \forall \varphi \in L^{2}(\mathbb{R}),
$$

then from (3.4) we easily conclude $u_{J}=P_{J} u$. From the point of view of an application to the problem (1.1), the important property of Shannon wavelets is the compactness of their support in the frequency space. Indeed, since

$$
\widehat{\psi}_{j, k}(\xi)=2^{-j / 2} e^{-i 2^{-j} k \xi} \widehat{\psi}\left(2^{-j} \xi\right), \quad \widehat{\phi}_{j, k}(\xi)=2^{-j / 2} e^{-i 2^{-j} k \xi} \widehat{\phi}\left(2^{-j} \xi\right),
$$

it follows that for any $k \in \mathbb{Z}$

$$
\operatorname{supp}\left(\widehat{\psi}_{j, k}\right)=\left\{\xi: \pi 2^{j} \leq|\xi| \leq \pi 2^{j+1}\right\}, \quad \operatorname{supp}\left(\widehat{\phi}_{j, k}\right)=\left\{\xi:|\xi| \leq \pi 2^{j}\right\}
$$


From (3.9), $P_{J}$ can be seen as a low-pass filter. The frequencies with greater than $\pi 2^{J+1}$ are filtered away.

Theorem 3.1. If $u(r, t)$ is the solution of problem (1.1) satisfying the condition $\|u(R, \cdot)\|_{p} \leq E$, then for any fixed $r \in\left(r_{1}, R\right]$

$$
\left\|u(r, \cdot)-P_{J} u(r, \cdot)\right\| \leq c_{3}^{-1}\left(2^{J+1}\right)^{-p} e^{(r-R) \sqrt{(1 / 2) \pi 2^{J}}} E .
$$

Proof. From (3.9), we have

$$
\begin{gathered}
u(r, \cdot)=\sum_{\lambda}\left\langle u(r, \cdot), \Psi_{\curlywedge}\right\rangle \Psi_{\lambda}, \\
P_{J} u(r, \cdot)=\sum_{\lambda \in \tilde{I}_{J}}\left\langle u(r, \cdot), \Psi_{\curlywedge}\right\rangle \Psi_{\lambda} .
\end{gathered}
$$

Due to Parseval relation and (2.8), (2.19), and (2.1), there holds

$$
\begin{aligned}
& \left\|u(r, \cdot)-P_{J} u(r, \cdot)\right\|=\left\|\widehat{u}(r, \cdot)-\widehat{P_{J} u}(r, \cdot)\right\| \\
& =\left\|\sum_{\lambda \in \tilde{I}}\left\langle\widehat{u}, \widehat{\Psi}_{\curlywedge}\right\rangle \widehat{\Psi}_{\lambda}-\sum_{\lambda \in \tilde{I}_{J}}\left\langle\widehat{u}, \widehat{\Psi}_{\lambda}\right\rangle \widehat{\Psi}_{\curlywedge}\right\| \\
& =\left\|\sum_{\lambda \in \tilde{I}_{j \supset J+1}}\left\langle\widehat{u}, \widehat{\Psi}_{\lambda}\right\rangle \widehat{\Psi}_{\lambda}\right\| \\
& =\left\|\sum_{\lambda \in \tilde{I}_{\geq \geq J+1}}\left\langle\left(\frac{I_{0}(\sqrt{i(\cdot)} r)}{I_{0}\left(\sqrt{i(\cdot)} r_{1}\right)}\right) \hat{g}(\cdot), \widehat{\Psi}_{\lambda}\right\rangle \widehat{\Psi}_{\lambda}\right\| \\
& =\left\|\sum_{\lambda \in \tilde{I}_{j \geq J+1}}\left\langle\left(\frac{I_{0}(\sqrt{i(\cdot)} r)}{I_{0}(\sqrt{i(\cdot)} R)}\right) \widehat{f}(\cdot), \widehat{\Psi}_{\lambda}\right\rangle \widehat{\Psi}_{\lambda}\right\| \\
& \leq \sup _{\pi 2^{J} \leq|\xi| \leq \pi 2^{J+1}}\left[|\xi|^{-p}\left|\frac{I_{0}(\sqrt{i \xi} r)}{I_{0}(\sqrt{i \xi} R)}\right|\right]\left\|\sum_{\lambda \in \tilde{I}_{j \geq J+1}}\left\langle\left(1+(\cdot)^{2}\right)^{p / 2} \widehat{f}(\cdot), \widehat{\Psi}_{\lambda}\right\rangle \widehat{\Psi}_{\lambda}\right\| \\
& \leq \sup _{\pi 2^{J} \leq|\xi| \leq \pi 2^{J+1}} c_{4}|\xi|^{-p} e^{(r-R) \sqrt{|\xi| / 2}} E \leq c_{4}\left(2^{J+1}\right)^{-p} e^{(r-R) \sqrt{(1 / 2) \pi 2^{\prime}}} E .
\end{aligned}
$$

Hence the conclusion of Theorem3.1 is proved.

\section{Error Estimates via Dual Least Squares Method Approximation}

Before giving error estimates, we present firstly subspaces $Y_{j}$. According to $K^{*} Y_{j}=V_{j}$, the subspaces $Y_{j}$ are spanned by $\rho_{\lambda}, \lambda \in \tilde{I}_{J}$, where

$$
K^{*} \rho_{\lambda}=\Psi_{\lambda}, \quad k_{\curlywedge}=\left\|\rho_{\lambda}\right\|^{-1}, \quad y_{\lambda}=\frac{\rho_{\lambda}}{\left\|\rho_{\lambda}\right\|}=k_{\lambda} \rho_{\lambda}
$$


$\rho_{\lambda}$ can be determined by solving the following parabolic equation (see Lemma 2.3):

$$
\begin{gathered}
-\frac{\partial U}{\partial t}=\Delta U=\frac{\partial^{2} U}{\partial r^{2}}+\frac{1}{r} \frac{\partial U}{\partial r}, \quad 0<r \leq R, t \geq 0, \\
U(r, 0)=0, \quad 0 \leq r \leq R, \\
U\left(r_{1}, t\right)=\Psi_{j, k}(t), \quad t \geq 0, \\
U(r, t) \text { bounded in } r=0, t>0 .
\end{gathered}
$$

Since supp $\widehat{\psi}_{j, k}$ is compact, the solution exists for any $t \in(0, \infty)$. Similarly the solution of the adjoint equation is unique. Therefore for a given $\Psi_{\lambda}, \rho_{\lambda}$ can be uniquely determined according to (4.2), furthermore

$$
\widehat{\rho}_{\lambda}=\frac{\overline{I_{0}(\sqrt{i \xi} r)}}{\overline{I_{0}\left(\sqrt{i \xi} r_{1}\right)}} \widehat{\Psi}_{\lambda}(\xi) \Longleftrightarrow \widehat{y}_{\lambda}=\frac{\overline{I_{0}(\sqrt{i \xi} r)}}{\overline{I_{0}\left(\sqrt{i \xi} r_{1}\right)}} k_{\lambda} \widehat{\Psi}_{\lambda}(\xi), \quad \lambda=\{j, k\} .
$$

The approximate solution for noisy data $g_{\delta}$ is explicitly given by

$$
P_{J} u^{\delta}(r, t)=u_{J}^{\delta}=\sum_{\lambda \in \tilde{I}_{J}}\left\langle u^{\delta}, \Psi_{\curlywedge}\right\rangle \Psi_{\lambda}=\sum_{\lambda \in \tilde{I}_{J}}\left\langle g_{\delta}, y_{\lambda}\right\rangle \frac{1}{k_{\lambda}} \Psi_{\lambda}
$$

Now we will devote to estimating the error $\left\|P_{J} u^{\delta}-P_{J} u\right\|$.

Theorem 4.1. If $g_{\delta}$ is noisy data satisfying the condition $\left\|g(\cdot)-g_{\delta}(\cdot)\right\| \leq \delta$, then for any fixed $r \in\left(r_{1}, R\right]$

$$
\left\|P_{J} u^{\delta}-P_{J} u\right\| \leq c_{4} e^{\left(r-r_{1}\right) \sqrt{(1 / 2) \pi 2^{J}}} \delta .
$$

Proof. From (4.3), we have $\widehat{y}_{\lambda}=\left(\overline{I_{0}(\sqrt{i \xi} r)} / \overline{I_{0}\left(\sqrt{i \xi} r_{1}\right)}\right) k_{\lambda} \widehat{\Psi}_{\lambda}$. Note that $P_{J} u^{\delta}$ given by (4.4), $P_{J} u$ given by (3.4) and (2.18), for $r_{1}<r \leq R$, there holds

$$
\begin{aligned}
\left\|P_{J} u^{\delta}(r, \cdot)-P_{J} u(r, \cdot)\right\| & =\left\|\sum_{\lambda \in \tilde{I}_{J}}\left\langle g_{\delta}-g, y_{\lambda}\right\rangle \frac{1}{k_{\lambda}} \Psi_{\lambda}\right\| \\
& =\left\|\sum_{\lambda \in \tilde{I}_{J}}\left\langle\widehat{g}_{\delta}-\widehat{g}, \widehat{y}_{\lambda}\right\rangle \frac{1}{k_{\lambda}} \widehat{\Psi}_{\lambda}\right\| \\
& =\left\|\sum_{\lambda \in \tilde{I}_{J}}\left\langle\widehat{g}_{\delta}-\widehat{g}, \frac{\overline{I_{0}(\sqrt{i(\cdot)} r)}}{\overline{I_{0}\left(\sqrt{i(\cdot)} r_{1}\right)}} k_{\lambda} \widehat{\Psi}_{\lambda}\right\rangle \frac{1}{k_{\lambda}} \widehat{\Psi}_{\lambda}\right\| \\
& \leq \sup _{\pi 2^{J-1} \leq|\xi| \leq \pi 2^{J}}\left|\frac{\overline{I_{0}(\sqrt{i \xi} r)}}{\overline{I_{0}\left(\sqrt{i \xi} r_{1}\right)}}\right| \cdot\left\|\sum_{\lambda \in \tilde{I}_{J}}\left\langle\widehat{g}_{\delta}-\widehat{g}, \widehat{\Psi}_{\lambda}\right\rangle \widehat{\Psi}_{\lambda}\right\|
\end{aligned}
$$


Boundary Value Problems

$$
\begin{aligned}
& \leq \sup _{\pi 2^{J-1} \leq|\xi| \leq \pi 2^{J}}\left|\frac{I_{0}(\sqrt{i \xi} r)}{I_{0}\left(\sqrt{i \xi} r_{1}\right)}\right| \cdot\left\|\widehat{P}_{J}\left(\widehat{g}_{\delta}-\widehat{g}\right)\right\| \\
& \leq \sup _{\pi 2^{J-1} \leq|\xi| \leq \pi 2^{J}}\left|\frac{I_{0}(\sqrt{i \xi} r)}{I_{0}\left(\sqrt{i \xi} r_{1}\right)}\right| \cdot \delta \\
& \leq c_{2} \sup _{\pi 2^{J-1} \leq \xi \xi \mid \leq \pi 2^{J}} e^{\left(r-r_{1}\right) \sqrt{|\xi| / 2}} \delta \\
& \leq c_{2} e^{\left(r-r_{1}\right) \sqrt{(1 / 2) \pi 2^{J}}} \delta .
\end{aligned}
$$

Hence the conclusion of Theorem 4.1 is proved.

The following is the main result of this paper.

Theorem 4.2. Let $u$ be the exact solution of (1.1) and let $P_{J} u^{\delta}$ be given by (4.4). If $\left\|g-g_{\delta}\right\| \leq \delta$ and $J=J(\delta)$ is such that

$$
J=\log _{2}\left[\frac{2}{\pi}\left(\frac{1}{R-r_{1}} \ln \left(\frac{E}{\delta}\left(\ln \frac{E}{\delta}\right)^{-2 p}\right)\right)^{2}\right],
$$

then for any fixed $r \in\left(r_{1}, R\right]$

$$
\begin{aligned}
& \left\|u(r, \cdot)-P_{J} u^{\delta}(r, \cdot)\right\| \\
& \quad \leq E^{1-(R-r) /\left(R-r_{1}\right)} \delta^{(R-r) /\left(R-r_{1}\right)}\left(\ln \frac{E}{\delta}\right)^{-2 p\left(1-(R-r) /\left(R-r_{1}\right)\right)}(C+o(1)) \quad \text { for } \delta \longrightarrow 0,
\end{aligned}
$$

where $C=\left(c_{4}\left(R-r_{1}\right)^{2 p}+c_{2}\right)$.

Proof. Combining Theorem 4.1 with Theorem 3.1, and noting the choice rule (4.7) of $J$, we can obtain

$$
\begin{aligned}
\| u(r, \cdot) & -P_{J} u^{\delta}(r, \cdot) \| \\
\leq & c_{4}\left(2^{J+1}\right)^{-p} e^{(r-R) \sqrt{(1 / 2) \pi 2^{J}}} E+c_{2} e^{\left(r-r_{1}\right) \sqrt{(1 / 2) \pi 2^{\prime}}} \delta \\
\leq & c_{4} E\left(R-r_{1}\right)^{2 p}\left(\ln \left(\frac{E}{\delta}\left(\ln \frac{E}{\delta}\right)^{-2 p}\right)\right)^{-2 p}\left(\frac{E}{\delta}\left(\ln \frac{E}{\delta}\right)^{-2 p}\right)^{(r-R) /\left(R-r_{1}\right)} \\
& +c_{2} \delta\left(\frac{E}{\delta}\left(\ln \frac{E}{\delta}\right)^{-2 p}\right)^{\left(r-r_{1}\right) /\left(R-r_{1}\right)} \\
\leq & E^{1-(R-r) /\left(R-r_{1}\right)} \delta^{(R-r) /\left(R-r_{1}\right)}\left(\ln \frac{E}{\delta}\right)^{-2 p\left(1-(R-r) /\left(R-r_{1}\right)\right)}\left\{\frac{c_{4}\left(\left(R-r_{1}\right) \ln (E / \delta)\right)^{2 p}}{\left(\ln \left((E / \delta)(\ln (E / \delta))^{-2 p}\right)\right)^{2 p}}+c_{2}\right\} .
\end{aligned}
$$


Note that

$$
\frac{\ln (E / \delta)}{\ln \left((E / \delta)(\ln (E / \delta))^{-2 p}\right)}=\frac{\ln (E / \delta)}{\ln (E / \delta)-2 p \ln (\ln (E / \delta))} \longrightarrow 1 \quad \text { for } \delta \longrightarrow 0
$$

thus, there holds, for $\delta \rightarrow 0$

$$
\begin{aligned}
& \left\|u(r, \cdot)-P_{J} u^{\delta}(r, \cdot)\right\| \\
& \quad \leq E^{1-(R-r) /\left(R-r_{1}\right)} \delta^{(R-r) /\left(R-r_{1}\right)}\left(\ln \frac{E}{\delta}\right)^{-2 p\left(1-(R-r) /\left(R-r_{1}\right)\right)}\left(c_{4}\left(R-r_{1}\right)^{2 p}+c_{2}+o(1)\right) .
\end{aligned}
$$

Hence the conclusion of Theorem 4.2 is proved.

Remark 4.3. (i) When $p=0$ and $r_{1}<r<R$, estimate (4.8) is a Hölder stability estimate given by

$$
\left\|u(r, \cdot)-P_{J} u^{\delta}(r, \cdot)\right\| \leq\left(c_{4}+c_{2}\right) E^{1-(R-r) /\left(R-r_{1}\right)} \delta^{(R-r) /\left(R-r_{1}\right)} .
$$

(ii) When $p>0, r_{1}<r<R$, estimate (4.8) is a logarithmical Hölder stability estimate.

(iii) When $p>0, r=R$, estimate (4.3) becomes

$$
\left\|u(R, \cdot)-u^{\delta}(R, \cdot)\right\| \leq E\left(\ln \frac{E}{\delta}\right)^{-2 p}\left(c_{4}\left(R-r_{1}\right)^{2 p}+c_{2}+o(1)\right) \longrightarrow 0 \quad \text { for } \delta \longrightarrow 0
$$

This is a logarithmical stability estimate.

Remark 4.4. In general, the a-priori bound $E$ is unknown in practice, in this case, with

$$
J=\log _{2}\left[\frac{2}{\pi}\left(\frac{1}{R-r_{1}} \ln \left(\frac{1}{\delta}\left(\ln \frac{1}{\delta}\right)^{-2 p}\right)\right)^{2}\right],
$$

then

$$
\left\|u(r, \cdot)-P_{J} u^{\delta}(r, \cdot)\right\| \leq \delta^{(R-r) /\left(R-r_{1}\right)}\left(\ln \frac{1}{\delta}\right)^{-2 p\left(1-(R-r) /\left(R-r_{1}\right)\right)}(\widetilde{C}+o(1)) \quad \text { for } \delta \longrightarrow 0,
$$

where $\tilde{C}=c_{4}\left(R-r_{1}\right)^{2 p} E+c_{2}$.

\section{Acknowledgments}

The work is supported by the National Natural Science Foundation of China (No. 10671085), the Hight-level Personnel fund of Henan University of Technology (2007BS028), and the 
Fundamental Research Fund for Natural Science of Education Department of Henan Province of China (No. 2009B110007).

\section{References}

[1] A. Carasso, "Determining surface temperatures from interior observations," SIAM Journal on Applied Mathematics, vol. 42, no. 3, pp. 558-574, 1982.

[2] C.-L. Fu, "Simplified Tikhonov and Fourier regularization methods on a general sideways parabolic equation," Journal of Computational and Applied Mathematics, vol. 167, no. 2, pp. 449-463, 2004.

[3] D. A. Murio, The Mollification Method and the Numerical Solution of Ill-Posed Problems, A WileyInterscience Publication, John Wiley \& Sons, New York, NY, USA, 1993.

[4] D. N. Hào and H.-J. Reinhardt, "On a sideways parabolic equation," Inverse Problems, vol. 13, no. 2, pp. 297-309, 1997.

[5] T. I. Seidman and L. Eldén, "An 'optimal filtering' method for the sideways heat equation," Inverse Problems, vol. 6, no. 4, pp. 681-696, 1990.

[6] L. Elden, "Solving the sideways heat equation by a method of lines," Journal of Heat Transfer, vol. 119, pp. 406-412, 1997.

[7] L. Eldén, F. Berntsson, and T. Regińska, "Wavelet and Fourier methods for solving the sideways heat equation," SIAM Journal on Scientific Computing, vol. 21, no. 6, pp. 2187-2205, 2000.

[8] T. Regińska and L. Eldén, "Solving the sideways heat equation by a wavelet-Galerkin method," Inverse Problems, vol. 13, no. 4, pp. 1093-1106, 1997.

[9] T. Regińska and L. Eldén, "Stability and convergence of the wavelet-Galerkin method for the sideways heat equation," Journal of Inverse and Ill-Posed Problems, vol. 8, no. 1, pp. 31-49, 2000.

[10] C.-L. Fu and C. Y. Qiu, "Wavelet and error estimation of surface heat flux," Journal of Computational and Applied Mathematics, vol. 150, no. 1, pp. 143-155, 2003.

[11] X.-T. Xiong and C.-L. Fu, "Determining surface temperature and heat flux by a wavelet dual least squares method," Journal of Computational and Applied Mathematics, vol. 201, no. 1, pp. 198-207, 2007.

[12] W. Cheng, C.-L. Fu, and Z. Qian, "A modified Tikhonov regularization method for a spherically symmetric three-dimensional inverse heat conduction problem," Mathematics and Computers in Simulation, vol. 75, no. 3-4, pp. 97-112, 2007.

[13] U. Tautenhahn, "Optimal stable approximations for the sideways heat equation," Journal of Inverse and Ill-Posed Problems, vol. 5, no. 3, pp. 287-307, 1997.

[14] H.-T. Chen, S.-Y. Lin, and L.-C. Fang, "Estimation of surface temperature in two-dimensionnal inverse heat conduction problems," International Journal of Heat and Mass Transfer, vol. 44, no. 8, pp. 1455-1463, 2001.

[15] H. R. Busby and D. M. Trujillo, "Numerical soluition to a two-dimensionnal inverse heat conduction problem," International Journal for Numerical Methods in Engineering, vol. 21, no. 2, pp. 349-359, 1985.

[16] O. M. Alifanov and N. V. Kerov, "Determination of external thermal load parameters by solving the two-dimensional inverse heat-conduction problem," Journal of Engineering Physics, vol. 41, no. 4, pp. 1049-1053, 1981.

[17] H. Louahlia-Gualous, P. K. Panday, and E. A. Artyukhin, "The inverse determination of the local heat transfer coefficients for nucleate boiling on horizontal cylinder," Journal of Heat Transfer, vol. 125, no. 1, pp. 1087-1095, 2003.

[18] Y. C. Hon and T. Wei, "A fundamental solution method for inverse heat conduction problem," Engineering Analysis with Boundary Elements, vol. 28, no. 5, pp. 489-495, 2004.

[19] A. Shidfar and R. Pourgholi, "Numerical approximation of solution of an inverse heat conduction problem based on Legendre polynomials," Applied Mathematics and Computation, vol. 175, no. 2, pp. 1366-1374, 2006.

[20] T. Regińska, "Application of wavelet shrinkage to solving the sideways heat equation," BIT Numerical Mathematics, vol. 41, no. 5, pp. 1101-1110, 2001.

[21] M. Abramowitz and I. A. Stegun, Eds., Handbook of Mathematical Functions: With Formulas, Graphs, and Mathematical Tables, Dover, New York, NY, USA, 1972.

[22] J. R. Wang, "The multi-resolution method applied to the sideways heat equation," Journal of Mathematical Analysis and Applications, vol. 309, no. 2, pp. 661-673, 2005. 